\title{
Photonic Crystal Light Deflection Devices Using the Superprism Effect
}

\author{
Toshihiko Baba, Member, IEEE, and Masanori Nakamura
}

\begin{abstract}
The superprism effect allows wide-angle deflection of the light beam in a photonic crystal (PC) by a slight change of the wavelength or the incident angle. In this paper, we discuss such light deflection outside the $\mathrm{PC}$, which is expected when the output end of the $\mathrm{PC}$ is tilted against the input end. The analysis of the dispersion surfaces indicates a deflection angle of $\pm 50^{\circ}$ in a two-dimensional PC composed of triangular lattice airholes by changing the incident angle by $\pm 2^{\circ}$ or the wavelength by $\pm 2 \%$. Light deflections inside and outside the PC are numerically demonstrated by the finite difference time-domain method. It displays not only the main output beam but also many diffracted waves, which satisfy the wavevector conservation condition. These waves are sufficiently suppressed and an almost collimated output beam is realized by a flat interface.
\end{abstract}

Index Terms-FDTD, light deflection, photonic band, photonic crystal, superprism.

\section{INTRODUCTION}

$\mathbf{L}$ IGHT deflection devices are used in optoelectronic systems such as displays, sensors, and printers. Most present devices have sizes on the order of centimeters and operate mechanically. Micro-electro-mechanical system devices fabricated by planar technology and driven by the electrostatic force can be of millimeter to micrometer order. In these devices, however, there are exclusive restrictions for the deflection angle, speed, applied voltage, and the flexibility of motion. In this paper, we discuss the possibility of a photonic crystal (PC) light deflection device, which operates by another principle and allows a wide deflection angle.

For PCs, various applications utilizing the photonic bandgap or peculiar dispersion characteristics have been proposed and studied so far. The superprism effect is one of the peculiar dispersion characteristics, which can be applied to a wavelength multi/demultiplexer and a dispersion compensator [1], [2]. Using this effect, the light beam incident on a PC can be widely deflected inside the PC by a slight change of the wavelength or incident angle of light, as shown in Fig. 1. Let us consider not only the input end but also the output end of the PC, and let us ignore for simplicity peculiar light diffractions at these ends. Because of the reciprocity theorem, the output beam axis should be parallel to the incident beam axis, when the input and output ends are parallel. On the other hand, an output end tilted against the input end allows a large deflection angle outside the

Manuscript received August 31, 2001; revised March 22, 2002. This work was supported in part by the Ministry of Education, Science, Sports and Culture under Grant-in-Aid 10210203 and by the Japan Science and Technology Corporation under CREST 530-13.

The authors are with the Division of Electrical and Computer Engineering, Yokohama National University, Yokohama 240-8501, Japan.

Publisher Item Identifier S 0018-9197(02)05713-5.

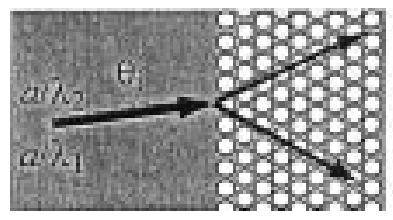

(a)

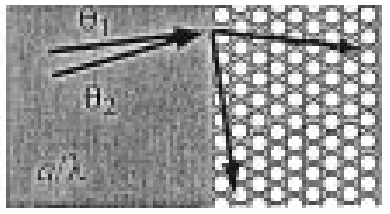

(b)
Fig. 1. Superprism effect in a PC against (a) different normalized frequencies and (b) different incident angles.

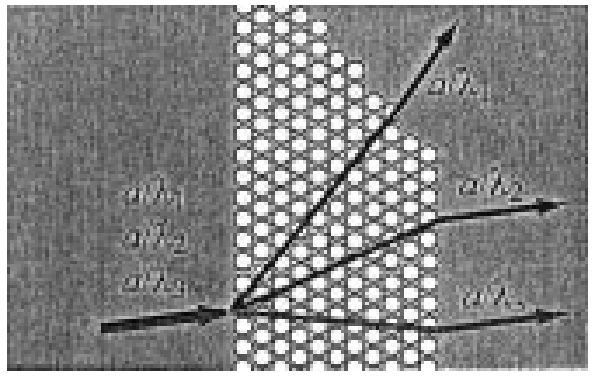

Fig. 2. Superprism effect against different normalized frequencies in the PC with two different angles of output end.

PC, as shown in Fig. 2. Thus, it acts as a light deflection device used with a tunable laser or another small angle deflection device.

Related to this type of device, various functions such as deflection, refraction, focusing, and birefringence have been studied for gratings in the 1980s [3], [4] and more recently for two-dimensional (2-D) PCs [5], [6]. Basically, such functions are estimated by the dispersion surface analysis established so far [7], [1]. However, a numerical simulation of the light beam is also necessary for the detailed design of a finite size structure including input and output ends [6]. It is particularly important for a superprism device in which a large refractive index contrast between the media is used to enhance the peculiarity of the dispersion characteristics. The aim of this study is to present some examples of high-performance light deflection devices designed by the dispersion surface and to discuss the actual beam propagation, degraded or improved by structural details.

In Section II, we explain the PC model and calculation methods used in this paper. In Section III, we review the dispersion surface analysis and confirm that the result agrees with simulations by the finite difference time-domain (FDTD) method. We also indicate that it is sometimes difficult to clearly demonstrate the superprism by the FDTD method because of the superprism effect itself and the small group velocity effect of the PC. In Section IV, we present two types of PC light deflection devices designed by dispersion surfaces. We estimate 


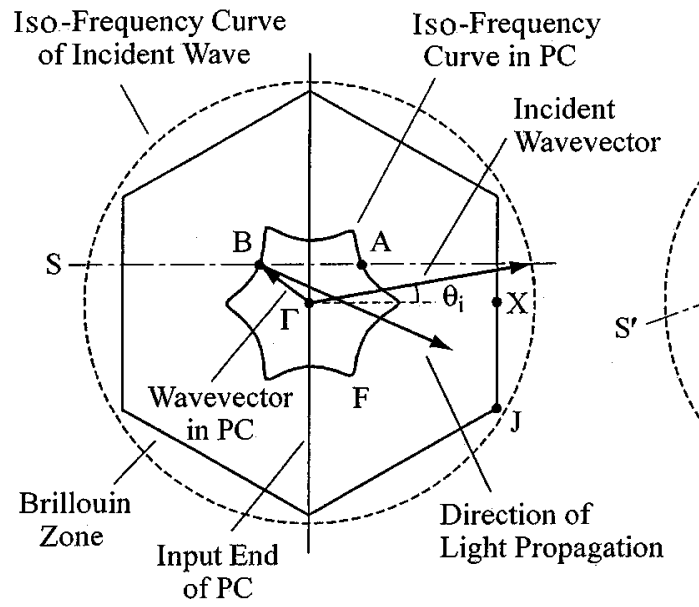

(a)

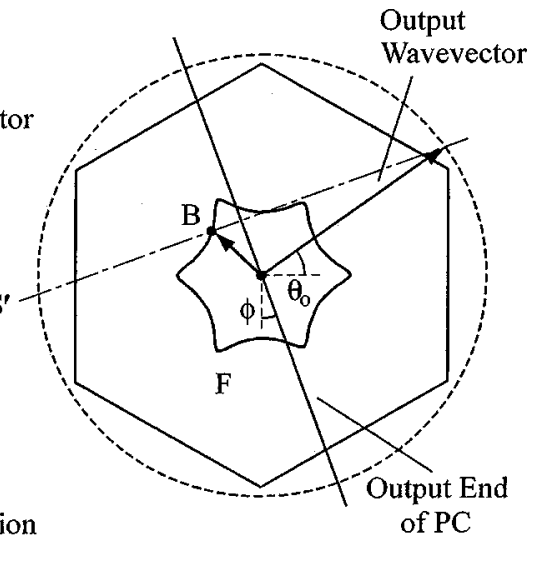

(b)

Fig. 3. Analysis of the deflection angle by using dispersion surfaces. An iso-frequency curve of $a / \lambda=0.64$ is calculated for a PC with $2 r / a=0.8637$. (a) Light deflection in the PC and (b) outside the PC.

a deflection angle of $\pm 50^{\circ}$ by arranging various tilt angles of the output end. In Section V, we show an FDTD simulation indicating that not only the main light beam but also unwanted diffracted waves are excited at the output end. Here, we propose the flat interface and demonstrate that diffracted waves can be suppressed perfectly by this interface when the tilt angle of the output end is less than $10^{\circ}$. Finally, we show the simulation of the light deflecting function for different frequencies.

\section{Models AND CALCUlations}

In this paper, we assume a 2-D PC composed of triangular lattice circular airholes. The height and refractive index of airholes are infinite and 1.0, respectively. The index of the background medium is 3.065. This value is the modal index of a silicon on insulator slab [8], which is often used in experiments. In the following, $2 r / a$ is used as a structural parameter, where $2 r$ is the diameter of airholes and a is the lattice pitch. The frequency of light is expressed as the normalized frequency $a / \lambda$, where $\lambda$ is the wavelength. The polarization of light is fixed so that the electric field vector lies inside the 2-D plane. A similar discussion will be possible for the orthogonal polarization. As shown in Fig. 1, the PC has an input end along the $\Gamma-J$ direction of the Brillouin zone, and the incident light beam enters from the background medium to the PC with angle $\theta_{i}$ measured from the normal to the input end. The output end is tilted against the input end by angle $\phi$. Here, the tilt angle is expressed by the step-like termination of the PC, as shown in Fig. 2. The output light beam is radiated out from the PC with angle $\theta_{\circ}$ also measured from the normal to the input end.

Dispersion surfaces of the PC are calculated by the plane-wave expansion method reported by Ho et al. [9] with 313 plane waves. In the FDTD method, 60 Yee cells describe pitch $a$. The diameter $2 r$ and pitch $a$ in the FDTD method are given so that the total area of one airhole described by Yee cells has the minimum difference to the airhole area assumed in the plane-wave expansion method. Berenger's perfectly matched layer absorbing boundary condition of 64 layers is used for the termination of the analytical space. As the incident wave, a continuous sinusoidal wave is excited for the magnetic field normal to the 2-D plane.
Note that, in all figures in this paper, the primary direction of light propagation is drawn to be from the left to the right. In displaying light intensity distributions calculated by the FDTD, field data are moderately thinned out for saving the graphical resources of the computer. This sometimes causes peculiar Moiré patterns, which are changed by the data-thinning ratio. Therefore, we will use only the results for discussing the deflection angle of light beam, not details of field profiles, phases, etc.

\section{SUPERPRISM EFFECT}

Fig. 3(a) shows an example of the iso-frequency curve $\mathrm{F}$ on a dispersion surface. By using this, the deflection angle of THE light beam inside the $\mathrm{PC}$ is predicted by the following dispersion surface analysis. First, we draw line $\mathrm{S}$ indicating the conservation of the tangential component of the incident wavevector at the input end. The vector from the $\Gamma$ point to the cross point of $S$ and $\mathrm{F}$ is the wavevector in the PC. The gradient of the dispersion surface at this cross point corresponds to the direction of light propagation. There are no less than two symmetric cross points $\mathrm{A}$ and B. One of them can be determined by considering the direction of light propagation. Around the normalized frequency $a / \lambda=0.64$ assumed in Fig. 3(a), the slope of the dispersion curve is negative against the length of the wavevector, i.e., the wavenumber. If one considers the forward propagating wave, point B should be selected, which gives the negative normal component of the wavevector but the positive slope of the dispersion surface.

Fig. 4 shows the light intensity distribution simulated by the FDTD method. Arrows indicate directions of light deflection predicted by the dispersion surface. The light beam simulated by the FDTD almost agrees with this prediction. A small difference may be caused by the digital error in the FDTD method. The light intensity inside the PC depends on the normalized frequency $a / \lambda$, but is $40 \%-50 \%$ of that before incidence. This decrease is caused by the reflection at the input end. Since the iso-frequency curve at $a / \lambda=0.55$ is relatively flat, light propagating in the PC is highly collimated and the intensity along its propagating direction is almost constant. Since the curve at $a / \lambda=0.61$ has four cross points with line $\mathrm{S}$, two different deflection angles are predicted. However, the intensity is concen- 


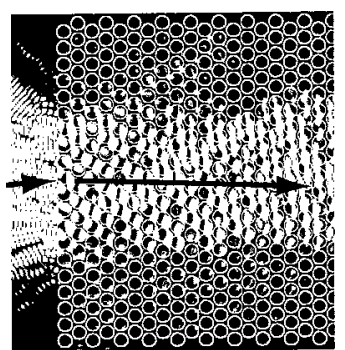

(a)

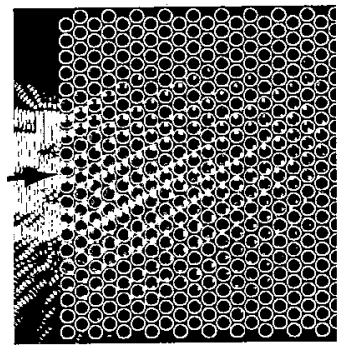

(c)

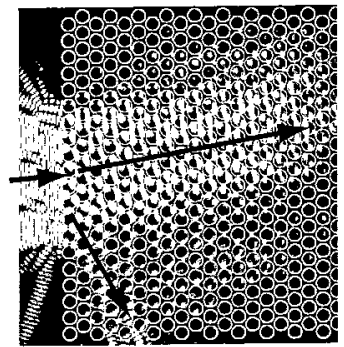

(b)

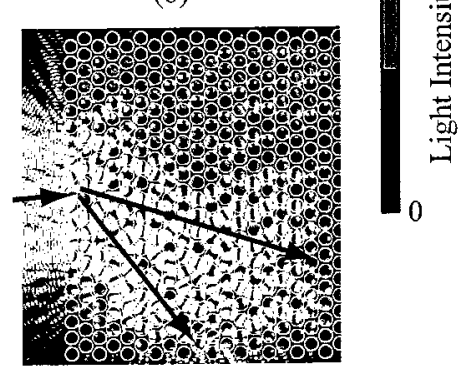

(d)
Fig. 4. Light intensity distributions calculated against normalized frequencies $a / \lambda$ of (a) 0.55 , (b) 0.61 , (c) 0.65 , and (d) 0.70 by the FDTD method. Open circles are added to indicate the position of airholes. $2 \mathrm{r} / a=0.8637$ and $\theta_{i}=$ $5^{\circ}$

trated in the main beam, and the intensity along the propagating direction is kept almost constant. At $a / \lambda=0.65$, the cross point cannot be defined clearly so that a sufficient light intensity is not observed in the PC. At $a / \lambda=0.70$, the interval between the curves is wide. This means a small group velocity inside the $\mathrm{PC}$. Therefore, the reflection at the input end is remarkable and only $<30 \%$ of light enters the PC. In addition, the intensity decays gradually along the propagating direction, since the light beam largely expands as it propagates.

In this FDTD calculation, the incident beam width was determined to be $8.3 a$ due to the restriction of the total size of the analytical space. Since the normalized frequency range focused on in this paper is $a / \lambda=0.5-0.8$, the beamwidth is $4 \lambda-6 \lambda$. This corresponds to $\sim 10$ times the diffraction limit in vacuum $(\sim 30$ times that in the background medium). However, the incident beam still includes angled plane-wave components to some extent. The superprism effect and the small group velocity effect scatter these components to various directions, expand the propagating beam, and generating some irregular beams. Actually, however, it is easy to expand the incident beamwidth to, for example, 100 times the diffraction limit and reduce such angled components in experiments. Therefore, we think that the beam quality and the power efficiency will not be actual problems. The reflection loss is another problem to be managed. Regarding this, we reported some structural modifications of the input end, which reduce the reflection to a negligible order in another paper [10].

\section{DeVICE DESIGN By Dispersion SuRfaCeS}

Fig. 3(b) shows the analysis of the deflection of the output beam, when the output end of the PC is tilted by angle $\phi$. The light propagating in the $\mathrm{PC}$ reaches the output end without losing the wavevector excited at the input end. The deflection angle can be determined by considering the conservation condition of the wavevector at the output end. In a manner similar to that at the
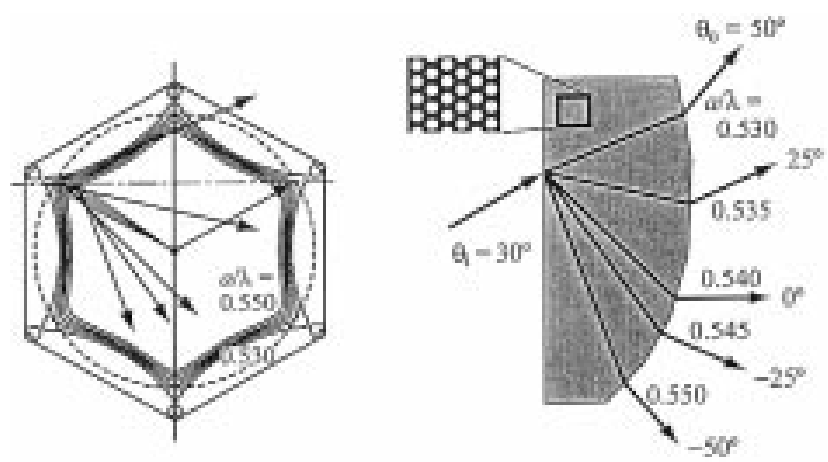

(a)
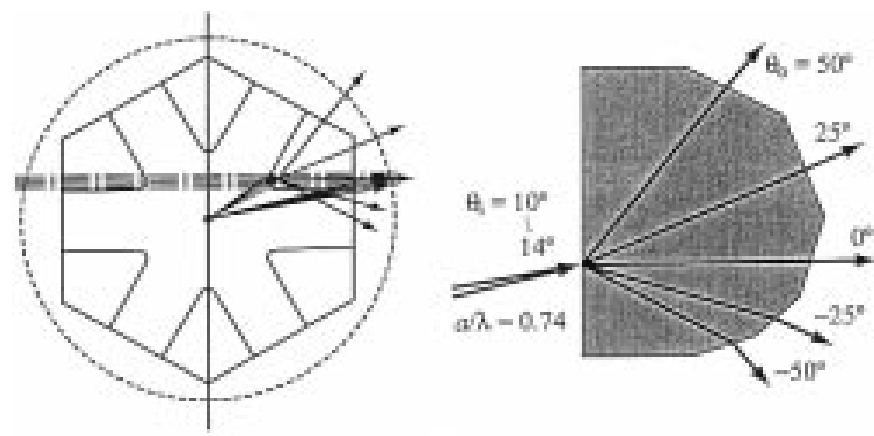

(b)

Fig. 5. PC light deflection devices designed by dispersion surfaces. (a) Device operated by a slight change of normalized frequency and (b) that by a slight change of incident angle. The left side shows the analysis of the deflection angle in the PC and the right side shows the profile of the designed device.

input end, the cross point of line $S^{\prime}$ and the iso-frequency curve of the output beam in the background medium is investigated. Since $S^{\prime}$ is changed by angle $\phi$, the deflection outside the PC can be controlled by angle $\phi$. For a wide deflection by a slight change of frequency, an iso-frequency curve should be selected, which shows a rapid change in the dispersion characteristic. On the other hand, for a wide deflection by a slight change of the incident angle, an iso-frequency curve with a complex shape should be selected. Fig. 5(a) and (b) shows examples of a designed device. The change of the normalized frequency $a / \lambda$ with wavenumbers is negative when $a / \lambda=0.530-0.550$ assumed in Fig. 5(a), while it is positive at $a / \lambda=0.74$ [assumed in Fig. 5(b)]. The maximum deflection angle of $\pm 50^{\circ}$ is designed by considering these properties. The device in Fig. 5(a) allows such a wide deflection by the combination of $\phi=-43^{\circ} \mp 10^{\circ}$ and a $\pm 2 \%$ change of the normalized frequency. The device in Fig. 5(b) allows this by $\phi=$ $-72^{\circ} \mp 63^{\circ}$ and $\mathrm{a} \pm 2^{\circ}$ change of the incident angle.

We designed these devices so that they exhibit a value of $\theta_{\circ}$ as large as possible. However, a remarkable deflection generally causes many diffracted waves of the output beam, as discussed in Section V. Actual light deflection devices are not always required to have such a large $\theta_{o}$. If the number of resolution points is important, a dispersion surface that moderately suppresses the superprism effect should be selected. We will discuss this issue elsewhere [11].

\section{Device Simulation by the FDTD Method}

In Section IV, we simply estimated the light deflection using the wavevector conservation condition at the output end. 


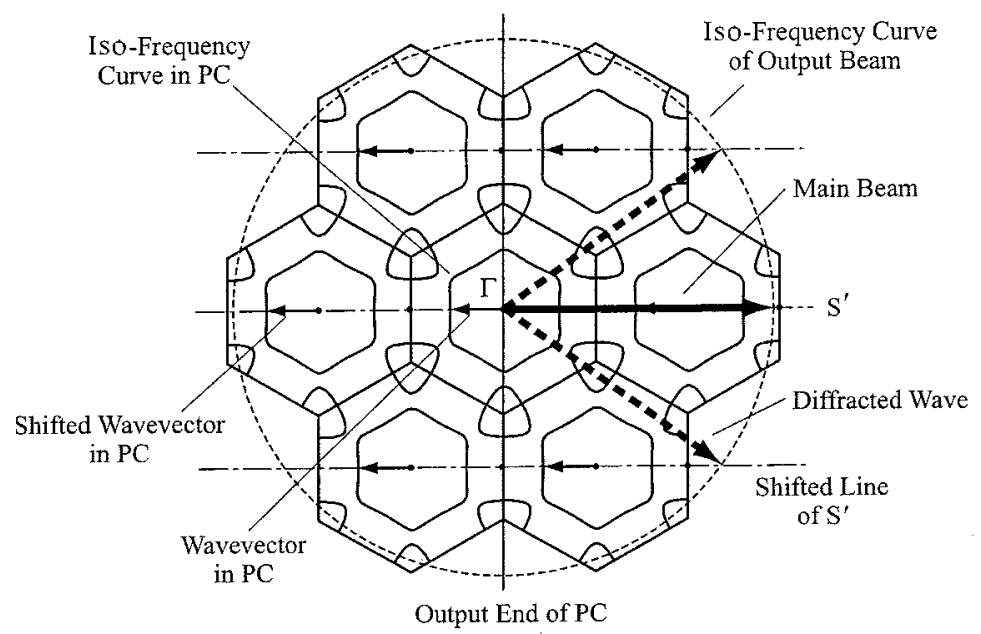

(a)

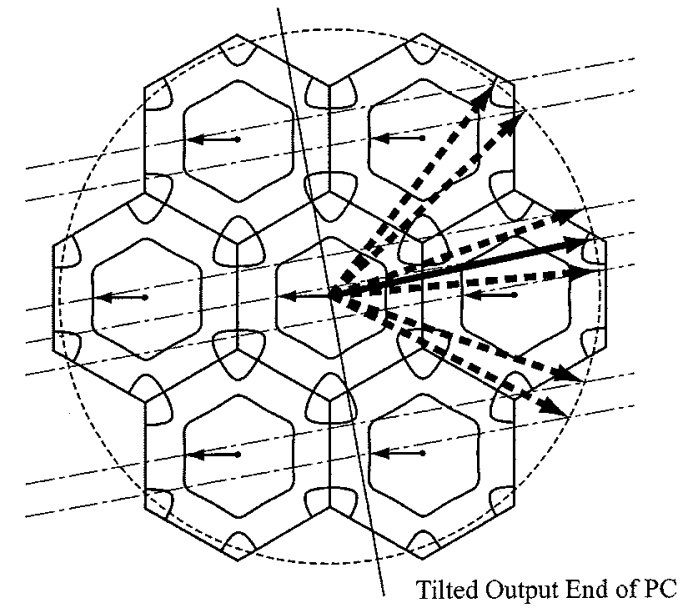

(b)

Fig. 6. Analysis of the deflection angle of the output beam. Line $S^{\prime}$ is shifted so that the wavevector conservation condition at the output end is explained. $2 r / a=0.8637, a / \lambda=0.55$, and $\theta_{i}=0^{\circ}$. (a) $\phi=0^{\circ}$. (b) $10^{\circ}$. Solid and dashed arrows indicate the main beam and diffracted waves, respectively.
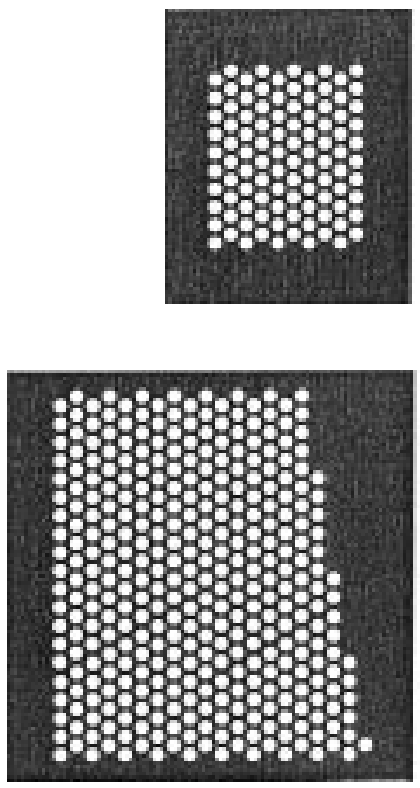

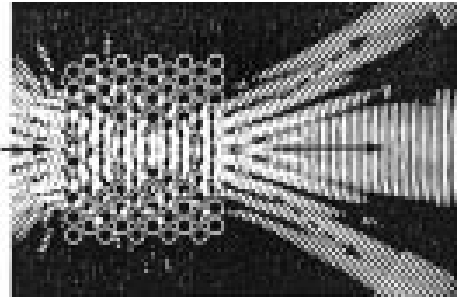

(a)

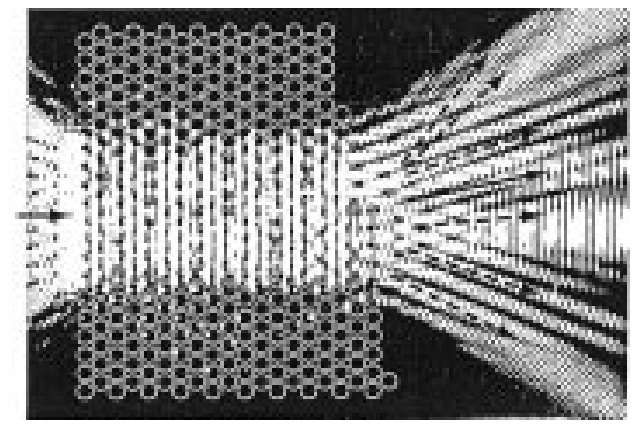

(b)

Fig. 7. Calculation models and light intensity distributions of output beam calculated by the FDTD method. Parameters are the same as for Fig. 6. Solid and dashed arrows indicate main beam and diffracted waves, respectively. Light intensity distribution of the output beam when (a) $\theta_{i}$ and $\phi$ are $0^{\circ}$ and (b) $\phi=10^{\circ}$.

However, the PC has an infinite number of reciprocal lattice vectors that shift the conservation condition. This actually excites many diffracted waves with various deflection angles. Fig. 6 explains the analysis of such waves. Since copies of line $S^{\prime}$ shifted by reciprocal lattice vectors generate many cross points with the iso-frequency curve of the output beam in the background medium, many extra wavevectors also satisfy the conservation condition. When $\theta_{i}$ and $\phi$ are $0^{\circ}$, three different wavevectors are predicted, as shown in Fig. 6(a). When the output end is tilted, many wavevectors with different $\theta_{\circ}$ are predicted, as shown in Fig. 6(b). These predictions are confirmed in the FDTD simulation. Fig. 7(a) shows the light intensity distribution of the output beam, when $\theta_{i}$ and $\phi$ are $0^{\circ}$. The solid arrow indicates the direction of deflection predicted from the unshifted line $S^{\prime}$, and dashed arrows indicate extra directions predicted from the shifted lines. Deflection angles $\theta_{0}$ simulated by the FDTD method almost agree with the prediction. Fig. 7(b) shows the output beam when $\phi=10^{\circ}$. In this case, six lines shifted by the smallest reciprocal lattice vectors are considered for the prediction of deflection angles. In a manner similar to Fig. 7(a), many diffracted waves are excited, and the deflection of the main beam is not clear. In general, diffracted waves become more dominant when the tilt angle $\phi$ is enlarged.

Various structural modifications of the output end are considered for clearer light deflection. The FDTD simulation indicates that the output end with the flat interface, which is composed of rectangular airholes connected by circular airholes, improves the problem. Fig. 8(a) shows the light intensity distribution for $\phi=$ $0^{\circ}$. The diffracted waves are remarkably suppressed and the main beam is well collimated. The intensity of the output beam is $~ 70 \%$ of that in the PC. This value indicates that most of the light not af- 

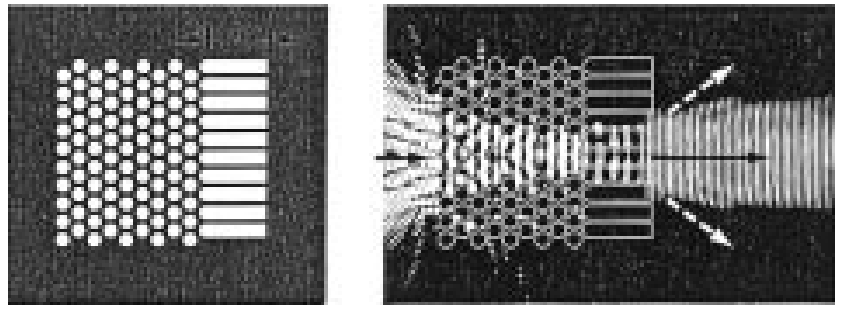

(a)
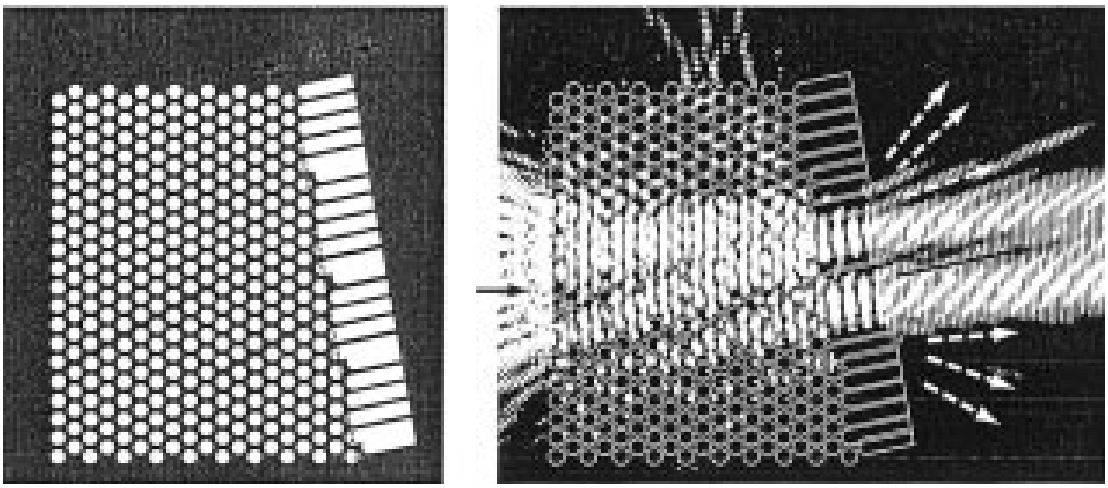

(b)

Fig. 8. Calculation models and light intensity distributions of the output beam from the PC with a flat interface calculated by the FDTD method. The parameters are the same as those in Fig. 6. Length $b$ of the rectangular airholes [the longest length for (b)] is four times the diameter $2 r$ of the circular airholes. Solid and dashed arrows indicate the main beam and diffracted waves, respectively. Light intensity distribution for (a) $\phi=0^{\circ}$ and (b) $\phi=10^{\circ}$.

fected by Fresnel reflection at the interface is collected into this main beam. The effectiveness of the flat interface is not sensitive to length $b$ of the rectangular airholes; an almost similar result is expected, when the PC is terminated by half circles. For these results, two reasons are considered. One is the less effective excitation of angled diffracted waves by the flat interface. The other is that the rectangular airholes change the shifting condition of line $\mathrm{S}^{\prime}$ and reduce the number of cross points between the shifted lines and the iso-frequency curve outside the PC. Fig. 8(b) shows the result for $\phi=10^{\circ}$. Here, the rectangular airholes are oriented in the direction of deflection predicted by the dispersion surface. Two rectangular airholes at each step-like arrangement of circular airholes are overlapping. The length of each rectangular airhole is adjusted so that the output end is on a straight line parallel to the output end of the PC. Therefore, the straight line is not necessarily normal to the orientation of the rectangular airholes. The simulated main beam is oriented in almost toward the same direction as that predicted by the dispersion surface. Even with the flat interface, the diffracted waves are gradually increased by a larger tilt angle $\phi$. To sufficiently suppress the diffracted waves, $\phi$ should be less than $10^{\circ}$.

Fig. 9(a)-(c) shows light intensity distributions when assuming three different tilt angles and three different normalized frequencies. Flat interfaces are designed in the same way as for Fig. 8(b). The light deflecting function is observed, which is well explained by dispersion curves. However, some noisy distributions and the remarkable expansion of the light beam are also observed. One reason is the Moire pattern, as explained in Section II. However, they too are evident at $a / \lambda=0.70$. This must be caused by the influence of the finite width of the incident beam and the small group velocity effect, as explained in Section III. On the other hand, Fig. 10(a)-(c) shows the light
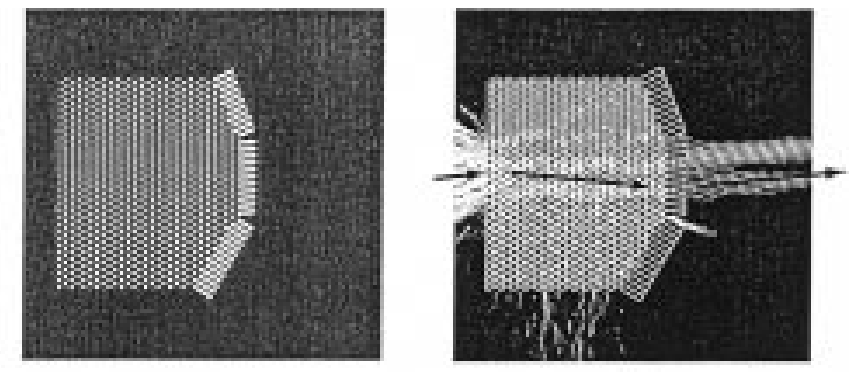

(a)

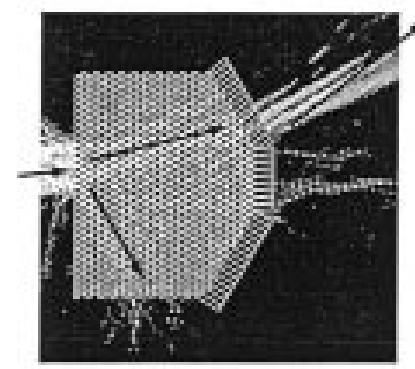

(b)

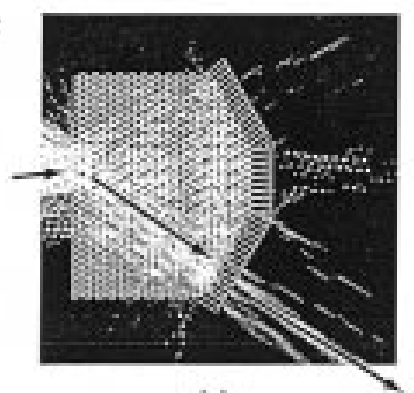

(c)
Fig. 9. Calculation model and light deflecting function of a PC with tilted flat interfaces simulated by the FDTD method. $2 r / a=0.8637, \theta_{i}=6^{\circ}$ and $\phi=$ $30^{\circ}, 0^{\circ}$ and $-30^{\circ}$. Normalized frequency $a / \lambda$ is (a) 0.55 , (b) 0.61 , and (c) 0.70

deflecting function, when air is assumed outside the output end of the PC. Here, three different normalized frequencies are selected so as to avoid the small group velocity effect. In this calculation, clearer light deflections are observed, as compared with those in Fig. 9(a)-(c). This seems to be due to the small iso-frequency curve outside the $\mathrm{PC}$, which reduces the cross points of the shifted lines and the iso-frequency curve. 

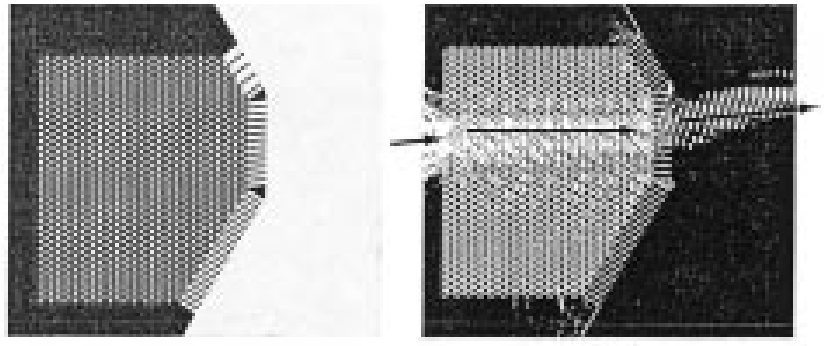

(a)

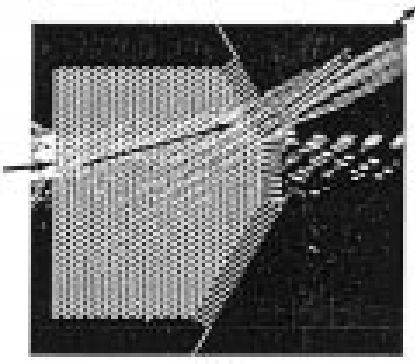

(b)

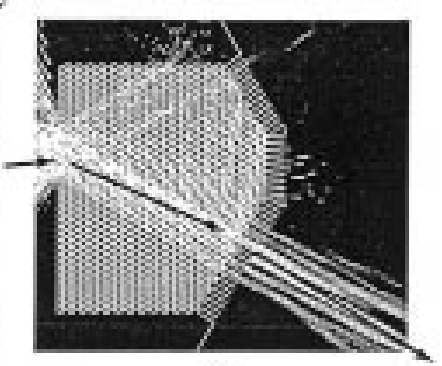

(c)
Fig. 10. Calculation model and light deflecting function of a $\mathrm{PC}$ with tilted flat interfaces simulated by the FDTD method. Structural parameters are the same as those for Fig. 9, except that air with an index of 1.0 is assumed outside the flat interfaces. Length $b$ is 4 times the diameter $2 r$ of the circular airholes. The normalized frequency $a / \lambda$ is (a) 0.56 , (b) 0.66 , and (c) 0.75 .

\section{CONCLUSION}

We proposed a PC light deflection device, which operates by the superprism effect and a tilt of the input and output ends of the PC. In the design of this device using dispersion surfaces, the deflection of the output beam of $\pm 50^{\circ}$ was predicted for a change of the frequency by $\pm 2 \%$ or for the incident angle by $\pm 2^{\circ}$. The superprism effect inside the PC, which was predicted by dispersion surfaces, agreed well with those simulated by the FDTD method. However, the FDTD simulation indicated that the quality of the output beam was seriously affected by diffracted waves. To suppress such waves, we proposed the flat interface at the output end of the PC, and confirmed its effectiveness in the simulation. We also confirmed the light deflecting function in such devices in the FDTD simulation.

A slight change of the incidence angle widely deflects the light beam in the PC, but it largely expands the output beam too. The number of resolution points of the incident light beam is not essentially improved. On the other hand, the frequency change can be a driving mechanism of deflecting a light beam. Therefore, a compact light deflection system will be possible by a combination of the PC light deflection device and a tunable laser.

\section{ACKNOWLEDGMENT}

The authors would like to thank Prof. Y. Kokubun, Yokohama National University, and Prof. K. Iga and Prof. F. Koyama, Tokyo Institute of Technology, for helpful suggestions.

\section{REFERENCES}

[1] H. Kosaka, T. Kawashima, A. Tomita, M. Notomi, T. Tamamura, T. Sato, and S. Kawakami, "Superprism phenomena in photonic crystals," Phys. Rev. B, vol. 58, pp. 10096-10099, 1998.

[2] — , "Photonic crystals for micro lightwave circuits using wavelength-dependent angular beam steering," Appl. Phys. Lett., vol. 74, pp. 1370-1372, 1999.

[3] P. St. J. Russell, "Novel thick-grating beam-squeezing device in $\mathrm{Ta}_{2} \mathrm{O}_{5}$ corrugated planar waveguide," Electron. Lett., vol. 20, pp. 72-73, 1984.

[4] R. Zengerle, "Light propagation in singly and doubly periodic planar waveguides," J. Mod. Opt., vol. 34, pp. 1589-1617, 1987.

[5] P. Halevi, A. A. Krokhin, and J. Arriaga, "Photonic crystal optics and homogenization of 2D periodic composites," Phys. Rev. Lett., vol. 82, pp. 719-722, 1999.

[6] B. Gralak, S. Enoch, and G. Tayeb, "Anomalous refractive properties of photonic crystals," J. Opt. Soc. Amer. A, vol. 17, pp. 1012-1020, 2000.

[7] P. St. J. Russell and T. B. Birks, "Bloch wave optics in photonic crystals: Physics and applications," in Photonic Band Gap Materials, C. M. Soukoulis, Ed. Boston, MA: Kluwer, 1996, pp. 71-92.

[8] T. Baba, N. Fukaya, and A. Motegi, "Clear correspondence between theoretical and experimental light propagation characteristics in photonic crystal waveguides," Electron. Lett., vol. 37, pp. 761-762, 2001.

[9] K. M. Ho, C. T. Chan, and C. M. Soukoulis, "Existence of a photonic band gap in periodic structures," Phys. Rev. Lett., vol. 65, pp. 3152-3155, 1990.

[10] T. Baba and D. Ohsaki, "Interfaces of photonic crystals for high efficiency light transmission," Jpn. J. Appl. Phys., vol. 40, pp. 5920-5924, 2001.

[11] T. Baba and T. Matsumoto, private communication, unpublished.

Toshihiko Baba (M'93) was born in Nagano Prefecture, Japan, in 1962. He received the B.E., M.E., and Ph.D. degrees from the Division of Electrical and Computer Engineering, Yokohama National University, Japan, in 1985, 1987, and 1990, respectively.

During his Ph.D. work, he was engaged in antiresonant reflecting optical waveguides (ARROWs) and integrated lightwave circuits. In 1990, he joined the Precision and Intelligence Laboratory, Tokyo Institute of Technology, Tokyo, Japan, as a Research Associate. In 1991, he reported the first calculation of spontaneous emission phenomena in microcavity vertical-cavity surface-emitting lasers (VCSELs). In 1993, he achieved the first room-temperature CW operation of a long-wavelength VCSEL. In 1994, he became an Associate Professor with Yokohama National University, Yokohama, Japan. From 1996 to 1997, he reported the lasing of a microdisk injection laser with the smallest cavity, the proposal and the first lasing of a deep grating distributed feedback laser, and fabrication and the first observation of light emission characteristics of photonic crystals. In 1999, he succeeded in demonstrating a photonic crystal waveguide operating at lightwave frequencies for the first time. Now his research interests are geared toward the spontaneous emission control in microdisk lasers and various device applications based on semiconductor photonic crystals.

Dr. Baba is a member of the Institute of Electronics, Information and Communication Engineers (IEICE), the Japan Society of Applied Physics, and the American Physics Society. He received the Niwa Memorial Prize in 1991, the Best Paper Award of Micro-Optic Conference in 1993 and 1999, the Paper Award and Academic Encouragement Award from IEICE in 1994, and the Marubun Research Encouragement Award in 2000.

Masanori Nakamura was born in Tokyo, Japan, in 1978. He received the B.E. degrees from the Division of Electrical and Computer Engineering, Yokohama National University, Japan, in 2001. He is currently working toward the M.E. degree at the Tokyo Institute of Technology, Tokyo, Japan.

Mr. Nakamura is a member of the Japan Society of Applied Physics. 